\title{
VARIABILITY OF THE EMISSION-LINE SPECTRUM OF THE NUCLEUS OF THE SEYFERT GALAXY NGC 7469
}

\author{
I. I. PRONIK \\ Crimean Astrophysical Observatory, Nauchny, U.S.S.R.
}

\begin{abstract}
It is shown that the emission lines of the NGC 7469 nucleus spectrum are variable. Time variability of hydrogen lines is less than 20 days. The $\mathrm{H} \alpha$ emission line is at the profile base about a factor of two narrower than the $\mathrm{H}_{\boldsymbol{\gamma}}$ line. Estimation of the density of hydrogen envelope, where Balmer lines wings were emitted, gives the value of about $10^{8}-10^{9} \mathrm{~cm}^{-3}$.
\end{abstract}

There are many reasons now for considering the nuclei of Seyfert galaxies as superstars with gaseous envelopes, emitting gas which can be compared with envelopes of peculiar stars or planetary nebulae.

During recent years it was shown that the emission line spectra of the nuclei of some Seyfert galaxies are variable (Andrillat and Souffrin, 1968, Khachikian and Weedman, 1971; Pronik and Chuvaev, 1972; Cherepashchuck and Lyutyi, 1973; Pronik, 1971, 1974a, b). This evidences that the physical conditions in the envelopes of Seyfert galaxy nuclei are variable. Investigations of such variations are important in the determination of the structure of Seyfert nuclei and their envelopes.

Variability of emission lines in the spectrum of the nucleus of Seyfert galaxy NGC 7469 was observed in 1971-1972. Twelve spectrograms with dispersion $380 \AA \mathrm{mm}^{-1}$ in the region 3700-6800 $\AA$ in 1971, September 22 and October 14, and 1972, January 7 and September 18 have been obtained at the prime focus of $2.6 \mathrm{~m}$ Shajn telescope with V. Pronik's high speed spectrograph.

The profiles of the hydrogen lines $\mathbf{H} \alpha-\mathbf{H} \delta$ averaged for each date are shown in Figure 1. One can see that:

(1) Profiles of Balmer lines on the same date differ from each other. The most peculiar among the Balmer lines is $\mathrm{H} \alpha$. Its profile for all observations is about a factor of two narrower than that of $\mathrm{H} \gamma-\mathrm{H} \delta$.

(2) Profiles of emission lines are variable with time. The most variations were observed in the profile of $\mathrm{H} \beta$. This profile was like that of $\mathrm{H} \alpha$ in 1972, January 7 whereas in 1972, September 18 - like those of $\mathrm{H} \gamma-\mathrm{H} \delta$.

(3) Time variation of hydrogen line profiles is less than 20 days.

Relative intensities of the brightest emission lines of the nucleus of NGC 7469 are shown in Table I. It summarizes the data from different sources. Intensities given in Table I corrected for interstellar absorption in the nucleus of Galaxy according to Wampler's data (Wampler, 1971) were used for the Balmer decrement calculations. The results are given in Figure 2, showing that the Balmer decrement is variable. Figures 3 and 4 show that relative intensities of emission lines correlate with the equivalent width of $\mathrm{H} \beta$ and the intensity of the continuum of the NGC 7469 nucleus. 


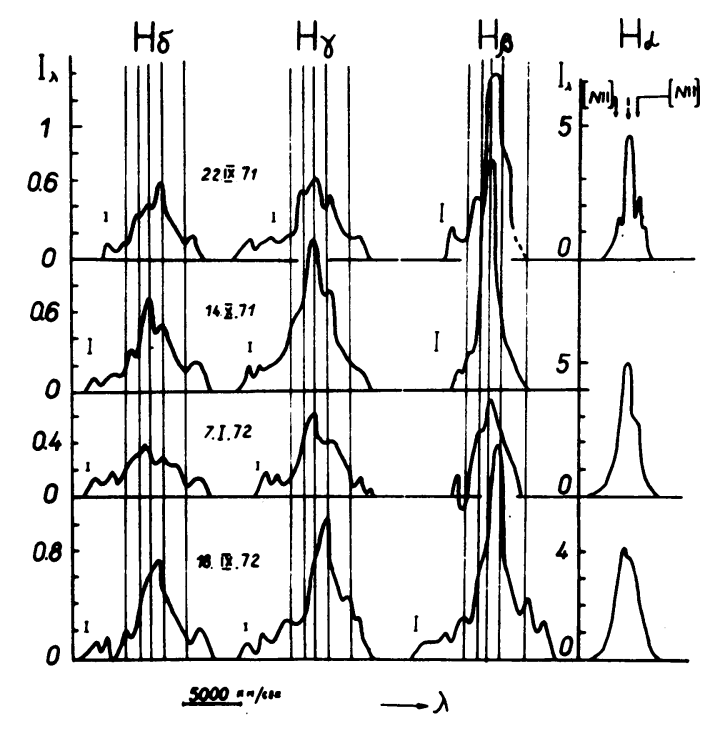

Fig. 1. Profiles of emission Balmer lines of NGC 7469 nucleus averaged for each date. Short vertical lines show the average square errors for each spectral line. Long vertical lines are drawn for easier comparison of the details of the spectral line profiles.

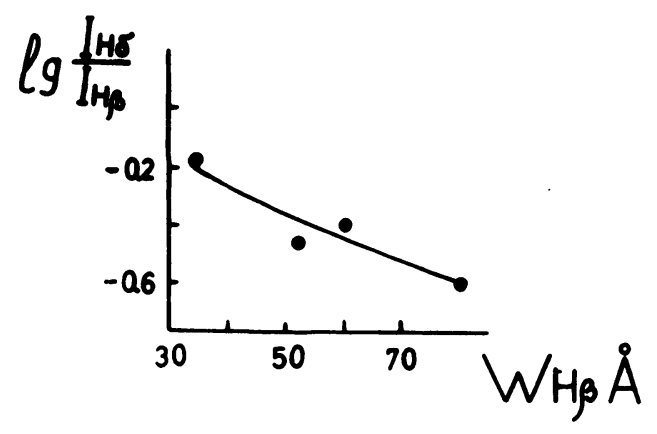

Fig. 2. Correlation between the relative line intensities $I_{\mathrm{H} \delta} / I_{\mathrm{H} \beta}$ and the equivalent width of the $\mathbf{H} \beta$ line $W_{\mathbf{H} \beta}$.

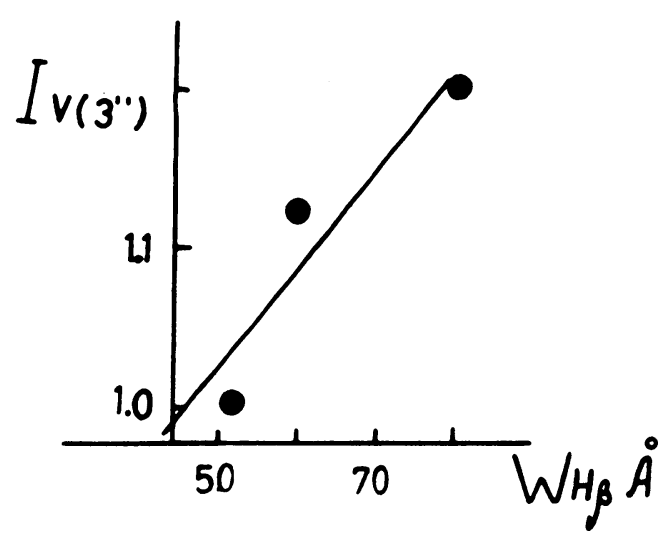

Fig. 3. Correlation between $W_{\mathrm{H} \beta}$ and intensity of the continuum $I_{v}\left(I_{v}\right.$ were calculated from data of Lyuty (1974)). 


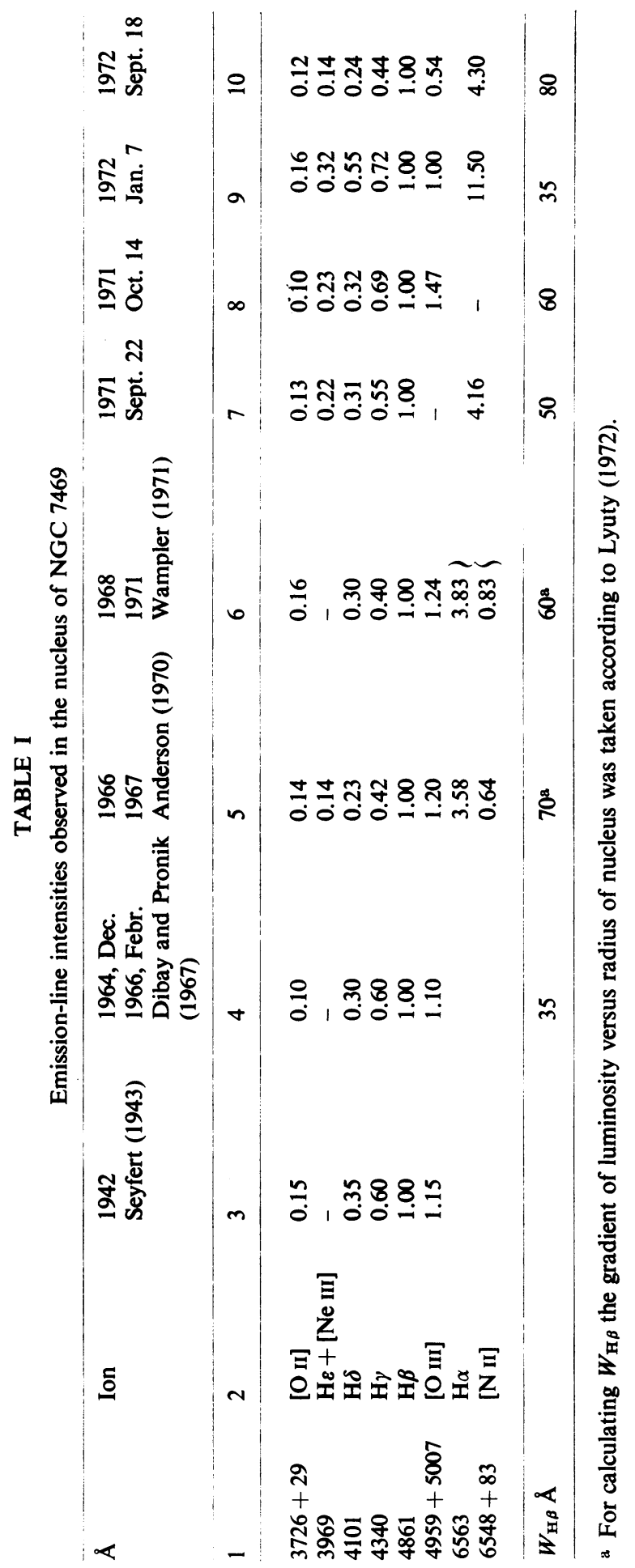




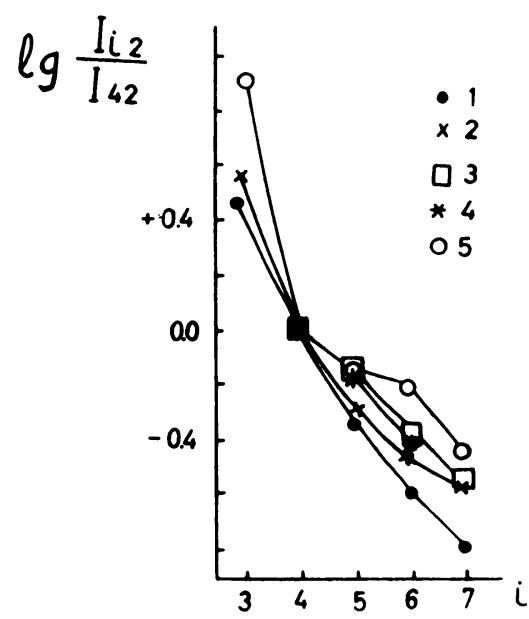

Fig. 4. Balmer decrements of the emission spectrum of the NGC 7469 nucleus:

$N 1$ according Anderson (1970) and our data 1972, September 18,

$N 2$ - 1971, September 22,

N 3 - 1971, October 14,

$N 4$ - according to Seyfert (1943) and Dibay and Pronik (1967),

N 5 - 1972, January 7.

The most common model for the nuclei envelopes of Seyfert galaxies is a thin or thick moving layer (Anderson, 1971; Ulrich, 1972). The theory of the Balmer decrements of such envelopes was given by Sobolev (1947), Boyarchuk (1966) and Gershberg (1974). Comparison of decrements of Figure 2 with the theoretical recombination decrements (Boyarchuk, 1966) allows us to obtain the parameters for the inner variable envelope for different dates. It was found that the inner part of the NGC 7469 nucleus envelope is optically thick. Table II gives its parameters for two dates: for 1972, January 7, when the Balmer decrement $I_{\mathrm{H} \beta}: I_{\mathrm{H} \gamma}: I_{\mathrm{H} \delta}: I_{\mathrm{H} \varepsilon}$ was the slopest and for 1972, September 18 - when it was the steepest of all the decrements observed. The columns of Table II are: (1) the date, (2) electron temperature $T_{e}$, (3) electron density $n_{\mathrm{e}}$, (4) size of the envelope $R,(5)$ mass $\mathbb{M}$ of that part of the envelope where the Balmer lines are variable.

TABLE II

Properties of the regions producing the variable Balmer emission

\begin{tabular}{lllll}
\hline Date & $T_{\mathrm{e}}^{\circ} \mathrm{K}$ & $n_{\mathrm{e}} \mathrm{cm}^{-3}$ & $R \mathrm{~cm}$ & $\overline{\mathfrak{M}} / \overline{\mathfrak{M}}_{\odot}$ \\
\hline 1972, Jan. 7 & $2 \times 10^{4}$ & $10^{9}$ & $1.6 \times 10^{16}$ & 17 \\
1972, Sept. 18 & $3 \times 10^{4}$ & $10^{8}$ & $5.0 \times 10^{16}$ & 25 \\
\hline
\end{tabular}

Thus our results show that physical conditions in the inner part of the hydrogen envelope of the nucleus of NGC 7469 are variable. In 1972, January 7 we observed the dense inner part of the envelope. In 1972, September 18 its $T_{\mathrm{e}}$ rose and we could observe the more rarefied outer part of the envelope. 
About a month earlier Lyuty observed a strong decrease in the $(U-B)$ of the NGC 7469 nucleus (Lyuty, 1974). This may be the cause of $T_{\mathrm{e}}$ rising in the envelope. Seyfert (1943), Dibay and Pronik (1967), Anderson (1970) and Wampler (1971) observed the NGC 7469 envelope to have different values of $T_{c}$ (Table I, Figure 2).

Our observations show that the inner variable envelope of the NGC 7469 nucleus is surrounded by a more extended and rarefied envelope, $10^{18}-10^{21} \mathrm{~cm}$ in size. This part of the envelope is more stable.

According to Dibay and Pronik, lines of [O II] $3727 \AA$ and $N_{1}+N_{2}[\mathrm{O}$ III] are emitted in the rarefied part of envelope, were $n_{\mathrm{e}}$ is equal to $10^{3}$ to $10^{6} \mathrm{~cm}^{-3}$. Our data show that the intensities of $3727 \AA$ and $N_{1}+N_{2}$ are also variable. For a detailed investigation of the character of such variations and physical conditions in this part of the envelope additional observations are needed.

The detailed results of this investigation will be published in the Astronomicheskij Zhurnal, 1975.

\section{Acknowledgements}

I am indebted to Drs R. Gershberg, A. Boyarchuk, V. Grinin and V. Pronik for discussions and to $T$. Nikulina for calculations and the drawing of the figures.

\section{References}

Anderson, K. S.: 1970, Astrophys. J. 162, 743.

Anderson, K. S.: 1971, Astrophys. J. 162, 449.

Andrillat, Y. and Souffrin, S.: 1968, Astrophys. Letters 1, 111.

Boyarchuk, A.: 1966, Izv. Krymsk. Astrofiz. Obs. 25, 45.

Cherepashchuk, A. and Lyuty, V.: 1973, Astrophys. Letters 13, 165.

Dibay, E. and Pronik, V.: 1967, Astron. Zh. 44, 952.

Gershberg, R.: 1974, Izv. Krymsk. Astrofiz. Obs. 50, 122.

Khachikian, E. Je. and Weedman, D. W.: 1971, Astrophys. J. 164, L109.

Lyuty, V.: 1972, Dissertation.

Lyuty, V.: 1974, in press.

Pronik, I.: 1971, Astron. Tsirk. U.S.S.R., No. 663.

Pronik, I.: 1974a, Astron. Zh. 51, 457.

Pronik, I.: 1974b, Astron. Zh., 51, 1204.

Pronik, V. and Chuvaev, K.: 1972, Astrofizika 8, 187.

Seyfert, C.: 1943, Astrophys. J. 97, 195.

Sobolev, V.: 1947, Moving Envelopes of Stars, Leningrad.

Ulrich, M.: 1972, Astrophys. J. 174, 483.

Wampler, E.: 1968, Astrophys. J. 154, L53.

Wampler, E.: 1971, Astrophys. J. 164, 1. 